\title{
A CASE OF CARCINOMA OF THE ANTRUM OF HIGHMORE.
}

By Frincis T. Morkix.

'I'He patient, a female aged 32, was admitted to st. Tincent's Hospital, 5th November, 1920, with a small tumour about the size of a hazel nut projecting above the right canine tooth.

She had several teeth extracted from time to time, those present showed moderate pyorrhea; six weeks previously the first upper molar was extracted. For a week afterward: the jaw was swollen and painful; this condition subsided and the tumour gradually appeared. She never suffered any pain and it was not tender on pressure. She had no epiphora or nasal discharge and no epistaxis.

6th November-The mucous membrane was reflected over the tumour, which was seen to be growing from the interior of the antrum; a piece was removed and sent to Dr. $O$ 'Farrell for examination.

The Pathologist's report is appended below.

8th November-With the assistance of Dr. P. J. Keogh I removed the maxilla. Preliminary ligature of the external carotid disclosed no enlarged gland.

The patient left hospital ten days after operation.

The tumour originated at the upper and pasterior wall of the antrum. X-Ray examination showed no involvement of bone by the growth, even where the anterior wall is perforated the bony trabecula were distinct. The growth apparently aiginated in the mucous membrane of the antrum, but did not show the character of columnar epithe. lioma.

Report on picce of tumour removed at first operation.

This specimen is about the size of a walnut and is of moderately firm consistency.

Sections cut show the following histological characters:--

The tumour consists of aberrant epithelium collected into masses of varying size and surrounded by fibrous tissue.

Th. epithelial celis are large and markedly vacuolated. 
giving rise to a net-work-like appearance. The nuclei are about one-fifth the size of the cells. Mitotic figures are fairly numerous.

The cells closely resemble squamous epithelium, but no cell-nests could be found.

In many places, particularly where the masses of epithelium are large, the tissue is necrotic.

The whole gection is more or less infiltrated with polynuclear leucostes, these being more numercus in the necrotic areas.

Right upper jaw romoved by operation.

The bone is practically intact, except where the tumow has perforated the anterior wall. A considerable amo:int of tumour substance was found in the nasal fossa, but this part of the new growth has become detached from the specimen; the site of invasion, however, can be seen. 